\title{
Clinical course and lung function change of idiopathic nonspecific interstitial
} pneumonia

\author{
I.N. Park*\#\#, Y. Jegal*\#\#, D.S. Kim*, K-H. Do\#, B. Yoo", T.S. Shim*, C-M. Lim*, \\ S.D. Lee*, Y. Koh*, W.S. Kim*, W.D. Kim*, S.J. Jang ${ }^{+}$, M. Kitaichi ${ }^{\S}$, \\ A.G. Nicholson ${ }^{f}$ and T.V. Colby**
}

ABSTRACT: Most studies of idiopathic nonspecific interstitial pneumonia (NSIP) have primarily studied mortality. In order to clarify the detailed outcome and prognostic markers in idiopathic NSIP, the clinical course with initial radiological and clinical features was analysed.

The clinical course of 83 patients who were classified with idiopathic NSIP (72 fibrotic, 11 cellular; 27 males and 56 females; mean \pm SD age $54.4 \pm 10.1$ yrs) was retrospectively analysed.

In fibrotic NSIP, $16(22 \%)$ patients died of NSIP-related causes with a median (range) follow-up of 53 (0.3-181) months. Despite the favourable survival (5-yr 74\%), patients with fibrotic NSIP were frequently hospitalised with recurrence rate of $36 \%$. Reduced forced vital capacity at 12 months was a predictor of mortality. On follow-up, lung function was improved or stable in $\sim 80 \%$ of the patients. The extent of consolidation and ground-glass opacity on initial highresolution computed tomography correlated significantly with serial changes of lung function, and the presence of honeycombing was a predictor of poor prognosis. During follow-up, eight (10\%) patients developed collagen vascular disease.

In conclusion, the overall prognosis of fibrotic nonspecific interstitial pneumonia was good; however, there were significant recurrences despite initial improvement and a subset of the patients did not respond to therapy. Some patients developed collagen vascular diseases at a later date.

KEYWORDS: Clinical course, fibrotic nonspecific interstitial pneumonia, pulmonary function test, recurrence

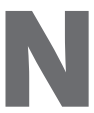
onspecific interstitial pneumonia (NSIP) is characterised histopathologically by temporally uniform interstitial pneumonia, in contrast to the temporal heterogeneity of usual interstitial pneumonia (UIP). NSIP was originally subcategorised into cellular NSIP (primarily inflammatory) and fibrotic NSIP (primarily fibrotic or mixed cellular and fibrotic) $[1,2]$. The prognosis of cellular NSIP is excellent $[3,4]$, in contrast to a median survival of fibrotic NSIP ranging 6-13.5 yrs [3, 5-9]. Although survival for idiopathic NSIP is well described, there are comparatively few data on clinical course and outcomes other than mortality, and its relationship with high-resolution computed tomography (HRCT) features or bronchoalveolar lavage (BAL) fluid differential cell counts.

Therefore, the purpose of the present study was to look specifically at parameters other than mortality, such as serial lung function changes, clinical course after completion of initial treatment, and development of collagen vascular diseases (CVDs) during follow-up, and to determine the prognostic value of baseline functional impairment, and BAL and HRCT features, in a cohort of patients who were diagnosed with idiopathic NSIP by initial surgical lung biopsy.

\section{METHODS}

\section{Subjects}

Between January 1991 and March 2006, 83 patients initially met the clinical, radiological and pathological criteria for idiopathic NSIP at Asan Medical Center, a tertiary referral center in Seoul, Korea. Eight patients developed CVDs during follow-up.

Biopsy was performed under the guidance of HRCT, either through open thoracotomy $(n=16)$

\section{AFFILIATIONS}

*Division of Pulmonary and Critical Care Medicine,

\#Dept of Radiology,

"Dept of Rheumatology, and +Dept of Pathology, University of Ulsan, College of Medicine, Asan Medical Center, Seoul, Korea, ${ }^{\S}$ Laboratory and Anatomic Pathology, National Hospital Organization Kinkichuo Chest Medical Center, Osaka, Japan,

${ }^{f}$ Dept of Histopathology, Royal Brompton Hospital, London, UK, and **Dept of Laboratory Medicine and Pathology, Mayo Clinic, Scottsdale, AZ, USA.

\#\#These authors contributed equally to the study as first authors.

\section{CORRESPONDENCE}

D.S. Kim

Division of Pulmonary and Critical Care Medicine

University of Ulsan College of

Medicine

Asan Medical Center

388-1 Pungnap-2dong

Songpa-gu

Seoul 138-736

Korea

Fax: 82230106968

E-mail: dskim@amc.seoul.kr

Received:

November 232007

Accepted after revision:

September 162008

STATEMENT OF INTEREST

None declared.

European Respiratory Journal

Print ISSN 0903-1936

Online ISSN 1399-3003 
or video-assisted thoracoscopic surgery $(n=67)$, and samples were taken from multiple sites in 70 patients (two lobes: 54 patients; three lobes: one patient; and two segments in one lobe: 15 patients), one site in nine patients, and in four patients who underwent biopsy at another hospital, no information about the number of specimens and sites selected was available.

Biopsy slides were reviewed independently by at least two pathologists and reclassified according to the American Thoracic Society (ATS)/European Respiratory Society consensus classification of interstitial pneumonia, as described in detail in a previous report [10], and only patients with NSIP pattern in all specimens were included for study. Diagnosis was not only on the basis of pathology but also with thorough clinical work-up and radiological evaluation. Patients with typical HRCT features of idiopathic pulmonary fibrosis (IPF), other interstitial lung disease (ILD) or significant amount of consolidation were excluded.

Patients with a history of drugs known to cause ILDs, occupational or other environmental exposures that could be implicated as a cause of ILD, or evidence of CVD as determined by thorough history taking, physical examination and serological tests at the time of diagnosis were also excluded [11]. Patients with positive serological tests without any clinical manifestations were included after rheumatological consultation. Most of the subjects in the present study have been included in previous reports $[5,10]$.

The study was approved by the institutional review board of Asan Medical Center.

\section{Data collection}

All clinical and laboratory data were collected retrospectively from medical records, and survival status up until October 2006 was obtained from telephone interviews and/or medical records. For each patient, the survival period was calculated from the date of lung biopsy. Patients were categorised as nonsmokers, current smokers, or ex-smokers (those who stopped smoking $\geqslant 3$ months before presentation). Recurrence was defined as aggravation of symptoms and lung function (and/or radiological abnormalities) after the complete discontinuation of treatment and the attending physician restarted treatment with prednisolone and/or cytotoxic agent.

\section{Pulmonary function tests}

Spirometry (Vmax22; Sensormedics, Yorba Linda, CA, USA), lung volumes by plethysmography (6200 Plethysmograph; Sensormedics) and diffusing capacity of the lung for carbon monoxide (DL,CO; Vmax229D; Sensormedics) were expressed as percentages of predicted values. Changes in pulmonary function tests (PFTs) were presented as the percentage change of initial value. Improvements and deteriorations were defined as $>10 \%$ changes in forced vital capacity (FVC) and total lung capacity (TLC), and $>15 \%$ changes in DL,CO [12]. Patients who did not demonstrate improvement or deterioration were considered stable. 6-min walk test was performed according to modified ATS guidelines [13].

\section{HRCT and BAL}

HRCT was performed with a HiSpeed Advantage Scanner (GE Medical Systems, Milwaukee, WI, USA) or a somatom Plus 4 scanner (Siemens Medical Systems, Erlangen, Germany). A pulmonary radiologist who was unaware of the clinical and functional findings reviewed the HRCT scans. Pulmonary parenchymal abnormalities were classified as consolidation, ground-glass opacity, reticular opacity and honeycombing. The extent of each abnormality was calculated as a mean extent of each lobe. The lingular division of left upper lobe was treated as a separate lobe. HRCT findings were categorised as reticular opacity dominant, ground-glass opacity dominant and mixed pattern. The dominant pattern was defined as one abnormality that doubly or more exceeded any other abnormal pattern. BAL was performed as previously described [14].

\section{Statistical methods}

Data are presented as mean \pm SD or median (range). Categorical data were compared using Chi-squared or Fisher's exact test, and continuous data were compared by unpaired t-test or the Mann-Whitney U-test. One-way repeated measures ANOVA was used to evaluate changes in FVC over time. Survival was evaluated using Kaplan-Meyer survival curves and the log rank test. Cox proportional hazards regression analysis was used to identify significant variables predicting survival status. $p<0.05$ was considered statistically significant (all tests were two-tailed).

\section{RESULTS}

\section{Baseline characteristics}

Of the 83 patients, 72 had fibrotic NSIP and 11 had cellular NSIP. Their baseline clinical and laboratory features are summarised in table 1 . Most patients presented with subacute onset of dyspnoea or nonproductive cough. However, two patients with fibrotic NSIP presented with an acute exacerbation and the diagnosis of NSIP was confirmed by surgical lung biopsy at that time. Between the cellular and fibrotic NSIP groups, there were no differences in the demographic features, smoking histories, PFTs, or resting arterial oxygen tension. A higher percentage of lymphocyte in BAL fluid was found in cellular NSIP.

\section{Initial therapy, recurrence and hospitalisation}

In cellular NSIP, all patients were treated and three $(27 \%)$ of them received more than one cycle of treatment. However, none of the patients with cellular NSIP was hospitalised due to the respiratory disorder during the study period.

In fibrotic NSIP, all but four patients were treated with corticosteroid alone or corticosteroids plus cytotoxic agent. Colchicine was given to one patient who had only mild symptoms. Among the three patients who were not treated, one had mild disease, one experienced a severe adverse effect to the treatment, and one died suddenly of unknown causes before treatment. The clinical courses after initial treatment in fibrotic NSIP group only are summarised in figure 1 . The duration of the initial therapy was $17.4 \pm 12.1$ months and all but one patient, who was treated for 9 months, were treated for $>1 \mathrm{yr}$, unless death occurred within $1 \mathrm{yr}$. The initial dose of prednisolone was $51.5 \pm 12.0 \mathrm{mg} \cdot \mathrm{day}^{-1}$. After $1 \mathrm{month}$, the prednisolone dose was slowly tapered. The interval from the start of treatment to $15 \mathrm{mg}$ was $5.7 \pm 3.7$ months. A total of 12 patients were treated with corticosteroid alone and the other 56 patients were treated with corticosteroid and cytotoxic agent. 


\begin{tabular}{|c|c|c|c|c|}
\hline \multirow[t]{2}{*}{ TABL } & \multirow[b]{2}{*}{ Total } & \multirow[b]{2}{*}{$\begin{array}{l}\text { Fibrotic } \\
\text { NSIP }\end{array}$} & \multirow[b]{2}{*}{$\begin{array}{l}\text { Cellular } \\
\text { NSIP }\end{array}$} & \multirow[b]{2}{*}{$\mathrm{p}$-value } \\
\hline & & & & \\
\hline Subjects & 83 & 72 & 11 & \\
\hline Age yrs & $54.4 \pm 10.1$ & $54.3 \pm 10.1$ & $55.4 \pm 10.8$ & 0.739 \\
\hline Males/females & $27 / 56$ & $23 / 49$ & $4 / 7$ & 0.743 \\
\hline Follow-up period months & $56.1 \pm 39.0$ & $58.0 \pm 40.9$ & $43.4 \pm 20.1$ & 0.071 \\
\hline Smoking never/ex/current & $57 / 13 / 13$ & $51 / 10 / 11$ & $6 / 3 / 2$ & 0.470 \\
\hline Duration of dyspnoea months & s $5.7 \pm 6.7$ & $5.9 \pm 7.0$ & $4.9 \pm 4.5$ & 0.666 \\
\hline Antinuclear antibody & 46/81 (57) & $38 / 70(54)$ & 8/11 (73) & 0.335 \\
\hline Rheumatoid factor & $14 / 81(17)$ & $13 / 70(19)$ & $1 / 11(9)$ & 0.679 \\
\hline Resting $\mathrm{Pa}, \mathrm{O}_{2} \mathrm{mmHg}$ & $82.5 \pm 14.4$ & $83.4 \pm 14.3$ & $77.2 \pm 15.0$ & 0.187 \\
\hline \multicolumn{5}{|l|}{ Initial PFTs } \\
\hline FVC \% pred & $63.6 \pm 14.6$ & $63.3 \pm 14.9$ & $66.8 \pm 13.1$ & 0.594 \\
\hline FEV1 \% pred & $71.3 \pm 17.1$ & $71.1 \pm 17.4$ & $72.5 \pm 16.1$ & 0.792 \\
\hline DL,CO \% pred & $58.9 \pm 19.1$ & $58.4 \pm 19.9$ & $62.3 \pm 13.2$ & 0.532 \\
\hline TLC \% pred & $72.9 \pm 19.1$ & $73.2 \pm 20.3$ & $71.1 \pm 9.5$ & 0.752 \\
\hline \multicolumn{5}{|l|}{ Initial BAL finding \% } \\
\hline Macrophage & $51.5 \pm 19.6$ & $55.5 \pm 17.9$ & $26.6 \pm 7.7$ & $<0.001$ \\
\hline Lymphocyte & $35.3 \pm 21.0$ & $30.7 \pm 18.0$ & $63.8 \pm 15.8$ & $<0.001$ \\
\hline Neutrophil & $10.3 \pm 11.4$ & $10.5 \pm 10.7$ & $8.9 \pm 16.2$ & 0.714 \\
\hline Eosinophil & $2.5 \pm 3.8$ & $2.6 \pm 4.0$ & $1.7 \pm 2.2$ & 0.520 \\
\hline T4/T8 ratio & $0.91 \pm 0.81$ & $1.00 \pm 0.84$ & $0.45 \pm 0.37$ & 0.077 \\
\hline \multicolumn{5}{|l|}{ Initial treatment } \\
\hline Initial dose of PD mg & $51.5 \pm 11.9$ & $51.5 \pm 12.0$ & $51.4 \pm 12.1$ & 0.980 \\
\hline Combination with $\mathrm{CX}$ & $62 / 79(78)$ & $56 / 68(82)$ & $6 / 11(54)$ & 0.052 \\
\hline Time to $15 \mathrm{mg}$ of PD months & $6.8 \pm 7.2$ & $5.7 \pm 3.7$ & $12.8 \pm 15.5$ & 0.187 \\
\hline Total duration months & $17.2 \pm 12.1$ & $17.4 \pm 12.1$ & $15.8 \pm 12.4$ & 0.706 \\
\hline
\end{tabular}

Data are presented as $\mathrm{n}$, mean $\pm \mathrm{SD}$ and $\mathrm{n} /$ total (\%). $\mathrm{Pa}, \mathrm{O}_{2}$ : arterial oxygen tension; PFT: pulmonary function test; FVC: forced vital capacity; \% pred: \% predicted; FEV1: forced expiratory volume in one second; $D L, C O$ : diffusion capacity of the lung for carbon monoxide; TLC: total lung capacity; BAL: bronchoalveolar lavage; PD: prednisolone; CX: cytotoxic agent. $1 \mathrm{mmHg}=0.133 \mathrm{kPa}$.

The dose of prednisolone was not different between corticosteroid alone group and combination group.

Among 55 patients who improved or were stable after the initial treatment, $20(36 \%)$ received more than one cycle of treatment due to recurrence. The interval from the completion of initial treatment to recurrence was $11.6 \pm 14.1$ months and the patients were treated with the same drugs used for the initial treatment, because they were effective. In detail, after the initial therapy $(n=68)$, FVC improved in $36(53 \%)$ patients, and in $25(69 \%)$ of these, FVC recovered to a normal range $(>80 \%$ pred). However, $13(36 \%)$ of the patients with initial improvement suffered a recurrence of disease and four patients ultimately died. Lung function was stable in 19 (28\%) patients after initial therapy and recurrence occurred in seven patients and disease-related death in three. There was no significant difference in baseline clinical, demographic, laboratory findings and treatment methods or duration between recurrence and no recurrence groups of fibrotic NSIP, except for the fact that there was a greater reduction of oxygen saturation during 6-min walk test in the recurrence group (table 2).
The remaining $13(19 \%)$ patients deteriorated progressively and nine $(69 \%)$ of them died within 5 yrs. There was no difference in the initial treatment between patients with initial deterioration and improved or stable patients (data not shown). According to the univariate Cox proportional hazard model, male sex, initial TLC, decline in FVC at 3 months and the presence of honeycombing on initial HRCT were predictors of progressive deterioration (see online supplementary material).

Among the fibrotic NSIP patients, 35 (49\% of the total) had a total of 69 all-cause hospitalisations during the study period, including 38 hospitalisations of 27 (38\% of the total) patients due to respiratory causes. The most common cause of hospitalisation was disease progression $(68 \%)$, with or without superimposed infection. There was no significant difference in baseline clinical and demographic features between hospitalised or nonhospitalised patients (data not shown). However, hospitalisation due to respiratory disorder was an independent risk factor for death $(\mathrm{p}=0.001)$.

\section{Changes in PFTs}

In fibrotic NSIP, the FVC of survivors and the dead from unrelated causes improved steadily. In contrast, the FVC of nonsurvivors deteriorated after the initial 6-month improvement (fig. 2). At 3 yrs after the diagnosis, 32 of 45 survivors had lung function tests performed and 20 (62\%) patients improved, 25\% were stable and 13\% had worsened. In cellular NSIP, all but two patients had improved lung function and $70 \%$ of them recovered to normal range (table 3 ).

\section{Initial HRCT features and BAL fluid findings}

The HRCT images at the time of diagnosis were available in 53 patients with fibrotic NSIP and 11 patients with cellular NSIP. No patient had a typical UIP pattern on initial HRCT. The prevalence and extent of individual HRCT patterns are summarised in table 4 . On the initial HRCT, there was no significant difference between fibrotic and cellular NSIP, except that a pattern of consolidation was both more frequently seen and seen to a larger extent in cellular NSIP.

In fibrotic NSIP patients, a negative correlation was found between the extent of radiological abnormalities on the initial HRCT and PFT (honeycombing with initial FVC, ground-glass opacity and reticulation with initial $D L, C O)$. Furthermore, the extent of consolidation on the initial HRCT was significantly correlated with the serial changes in FVC at 3, 6 and 12 months and $\mathrm{DL}, \mathrm{CO}$ at 12 months. The extent of ground-glass opacity was positively correlated with the serial changes in DL,CO at 6 and 12 months (table 5).

In fibrotic NSIP, 12 patients had ground-glass opacity dominant pattern, 12 patients reticular density dominant and 29 patients had mixed pattern. Among these groups no significant difference was found in the initial PFT or other baseline characteristics (see online supplementary material). Additionally, there was no significant difference in the serial pulmonary function change according to the dominant HRCT patterns (see online supplementary material).

\section{Survival}

All 83 patients were followed up to death or until October 2006. A total of 24 patients (21 fibrotic NSIP and 3 cellular 


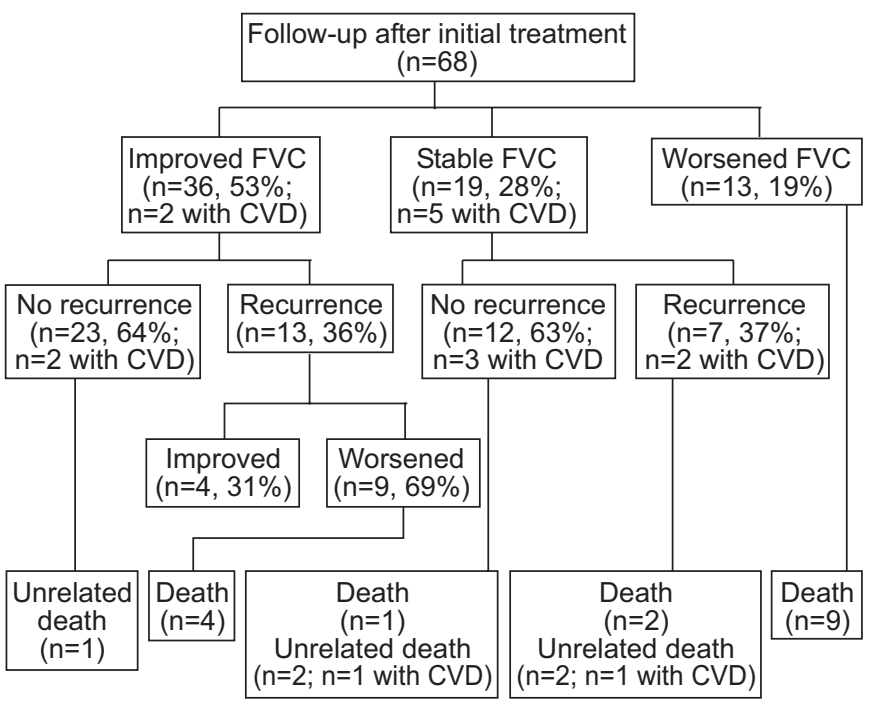

FIGURE 1. Clinical courses and final outcomes of the patients with fibrotic nonspecific interstitial pneumonia after initial treatment. Among the initial improvement group, forced vital capacity (FVC) improved to normal range $(>80 \%$ predicted) in 25 (69\%) patients. CVD: collagen vascular disease.

NSIP) died during a median follow-up of 53 months. Considering only disease-related death, 1-yr survival rate of fibrotic NSIP was 91\%, 2-yr survival was 85\%, and 5-yr survival rate was $74 \%$ (for all causes of death, 1-, 2- and 5-yr survival rate was $90 \%, 83 \%$ and $70 \%$, respectively). The cause of death in the majority (16 deaths, $76 \%$ ) of patients with fibrotic NSIP was related to respiratory disease: acute exacerbation (defined by the criteria of AKIRA et al. [15]) in two patients, disease progression with superimposed infection in four, disease progression without infection in nine, and disease progression with congestive heart failure and chronic renal failure in one. Another four patients died of unrelated disease (gastric cancer, laryngeal cancer, breast cancer and congestive heart failure) and one died from unknown causes. In patients with cellular NSIP, there was only one NSIP-related death (tuberculous myositis secondary to treatment); two patients died of unrelated disease (gastric cancer and unknown cause with uncontrolled back pain due to compression fracture).

Because the number of patients with cellular NSIP was small with only one disease-related death secondary to treatment in this group, only patients with fibrotic NSIP and disease-related mortality were used for survival analysis. On univariate Cox proportional hazard analysis, age, male sex, baseline PFT and hospitalisation for respiratory disorders were poor prognostic factors. Baseline 6-min walk test results were available in 39 patients. The degree of oxygen desaturation during the 6-min walk test was also a poor prognostic factor. With respect to the prognostic importance of three patterns in the initial HRCT, there was no disease-related death among the patients with ground-glass opacity dominance on HRCT. Because all but two patients with fibrotic NSIP had reticular opacity, Cox analysis was not performed for these subjects (table 6). Therefore, on initial HRCT, the presence of honeycombing was the only significant determinant of mortality in fibrotic NSIP on univariate Cox analysis.

\begin{tabular}{|c|c|c|c|c|}
\hline \multirow[t]{2}{*}{ TABLE 2} & \multicolumn{4}{|c|}{$\begin{array}{l}\text { Comparisons of clinical and demographic } \\
\text { features between fibrotic nonspecific interstitial } \\
\text { pneumonia with and without recurrence of } \\
\text { disease }\end{array}$} \\
\hline & & No recurrence & Recurrence & p-value \\
\hline \multicolumn{2}{|l|}{ Subjects } & 35 & 20 & \\
\hline \multicolumn{2}{|l|}{ Age yrs } & $52.4 \pm 10.7$ & $55.4 \pm 10.2$ & 0.314 \\
\hline \multicolumn{2}{|c|}{ Males/females } & 9/26 & $4 / 16$ & 0.749 \\
\hline \multicolumn{2}{|c|}{ Smoking never/ex/current } & $27 / 5 / 3$ & $16 / 1 / 3$ & 0.472 \\
\hline \multicolumn{2}{|c|}{ Duration of dyspnoea months } & $6.6 \pm 7.9$ & $5.4 \pm 7.0$ & 0.581 \\
\hline \multicolumn{2}{|c|}{ Antinuclear antibody } & $21 / 35(60.0)$ & 10/20 (50.0) & 0.575 \\
\hline \multicolumn{2}{|c|}{ Rheumatoid factor } & 7/34 (20.6) & $4 / 20(20.0)$ & 1.000 \\
\hline \multicolumn{5}{|l|}{ Initial PFTs } \\
\hline \multicolumn{2}{|l|}{ FVC \% pred } & $64.0 \pm 14.3$ & $64.8 \pm 18.1$ & 0.852 \\
\hline \multicolumn{2}{|c|}{ FEV $1 \%$ pred } & $72.0 \pm 16.7$ & $71.4 \pm 21.6$ & 0.901 \\
\hline \multicolumn{2}{|c|}{ DL,CO \% pred } & $58.4 \pm 19.3$ & $63.8 \pm 20.9$ & 0.348 \\
\hline \multicolumn{2}{|l|}{ TLC \% pred } & $74.1 \pm 16.0$ & $78.7 \pm 28.7$ & 0.472 \\
\hline \multicolumn{5}{|c|}{ Presence of lesions on initial HRCT } \\
\hline \multicolumn{2}{|c|}{ Ground-glass opacity } & $20(91)$ & $15(88)$ & 1.000 \\
\hline \multicolumn{2}{|c|}{ Consolidation } & $3(14)$ & $6(35)$ & 0.142 \\
\hline \multicolumn{2}{|c|}{ Reticular opacity } & $21(96)$ & $16(94)$ & 1.000 \\
\hline \multicolumn{2}{|c|}{ Honeycombing } & $1(5)$ & $2(12)$ & 0.570 \\
\hline \multicolumn{3}{|c|}{ Dominant pattern on initial HRCT } & & 0.683 \\
\hline \multicolumn{2}{|c|}{ Ground-glass opacity } & 5 & 6 & \\
\hline \multicolumn{2}{|c|}{ Reticular opacity } & 5 & 3 & \\
\hline \multicolumn{2}{|l|}{ Mixed } & 12 & 8 & \\
\hline \multicolumn{5}{|c|}{ Initial BAL findings \% } \\
\hline \multicolumn{2}{|c|}{ Macrophage } & $56.7 \pm 17.2$ & $55.5 \pm 18.9$ & 0.834 \\
\hline \multicolumn{2}{|c|}{ Lymphocyte } & $33.6 \pm 17.0$ & $26.6 \pm 18.2$ & 0.224 \\
\hline \multicolumn{2}{|l|}{ Neutrophil } & $7.6 \pm 5.8$ & $13.5 \pm 13.0$ & 0.099 \\
\hline \multicolumn{2}{|l|}{ Eosinophil } & $1.8 \pm 2.1$ & $2.7 \pm 3.4$ & 0.348 \\
\hline \multicolumn{2}{|l|}{ T4/T8 ratio } & $0.97 \pm 0.93$ & $0.91 \pm 0.63$ & 0.810 \\
\hline Initial 6-min v & alk test & & & \\
\hline Distance $\mathrm{m}$ & & $462.8 \pm 99.5$ & $440.1 \pm 142.4$ & 0.638 \\
\hline Change in $s$ & aturation \% & $6.9 \pm 6.6$ & $12.8 \pm 6.7$ & 0.048 \\
\hline Initial treatme & & & & \\
\hline Initial dose & f PD mg & $51.5 \pm 11.0$ & $50.5 \pm 12.9$ & 0.770 \\
\hline Combinatior & of $\mathrm{CX}$ & 28/35 (80) & $17 / 20(85)$ & 1.000 \\
\hline
\end{tabular}

Data are presented as $n$, mean \pm SD, $n /$ total (\%) or $n(\%)$. PFT: pulmonary function test; FVC: forced vital capacity; \% pred: \% predicted; FEV1: forced expiratory volume in one second; $D \mathrm{~L}, \mathrm{CO}$ : diffusion capacity of the lung for carbon monoxide TLC: total lung capacity; HRCT: high-resolution computed tomography; BAL: bronchoalveolar lavage; PD: prednisolone; CX: cytotoxic agent.

Mortality was substantially higher in those with $>10 \%$ deterioration in FVC 1 yr after diagnosis $(p=0.017$; see online supplementary material). However, the serial change in lung function at 6 months had no relationship with mortality $(p=0.276)$. Although there was no NSIP-related death in patients who later developed CVD, it was not a statistically significant predictor of mortality in univariate Cox analysis.

\section{Characteristics of the patients who developed CVD after the diagnosis of NSIP}

Among 83 patients with initial clinical and histological diagnosis of idiopathic NSIP, eight $(10 \%)$ patients (one cellular NSIP and seven fibrotic NSIP; one male and seven females; age 


\begin{tabular}{|c|c|c|c|c|c|c|c|c|c|c|c|}
\hline & \multirow[t]{2}{*}{ Subjects $n$} & \multicolumn{5}{|c|}{ Change in FVC } & \multicolumn{5}{|c|}{ Change in $D \mathrm{~L}, \mathrm{Co}$} \\
\hline & & $\%$ & $\Delta \mathrm{FVC}$ & Stable \% & $\%$ & $\Delta \mathrm{FVC}$ & $\%$ & $\Delta D \mathrm{~L}, \mathrm{CO}$ & Stable \% & $\%$ & $\Delta D \mathrm{~L}, \mathrm{CO}$ \\
\hline 6 months & 56 & 59 & $33 \pm 22$ & 25 & 16 & $19 \pm 6$ & 33 & $47 \pm 25$ & 50 & 17 & $27 \pm 9$ \\
\hline 12 months & 57 & 58 & $40 \pm 28$ & 28 & 14 & $20 \pm 8$ & 37 & $48 \pm 32$ & 48 & 15 & $28 \pm 12$ \\
\hline $2 \mathrm{yrs}$ & 41 & 58 & $48 \pm 30$ & 27 & 15 & $23 \pm 8$ & 46 & $45 \pm 34$ & 38 & 16 & $34 \pm 12$ \\
\hline $3 \mathrm{rs}$ & 32 & 62 & $46 \pm 31$ & 25 & 13 & $21 \pm 12$ & 53 & $49 \pm 41$ & 36 & 11 & $30 \pm 18$ \\
\hline \multicolumn{12}{|l|}{ Cellular NSIP } \\
\hline $3 \mathrm{yrs}$ & 6 & 67 & $68 \pm 62$ & 17 & 17 & 22 & 67 & $69 \pm 30$ & 33 & 0 & 0 \\
\hline
\end{tabular}

Data are presented as mean \pm SD, unless otherwise stated. $\Delta$ refers to the mean change from the initial forced vital capacity (FVC) or diffusion capacity of the lung for carbon monoxide $(D L, C O)$ value presented as \% predicted. Improved FVC: $>10 \%$ increase; worsened: $>10 \%$ decrease. Improved $D L, C O:>15 \%$ increase; worsened: $>15 \%$ decrease. NSIP: nonspecific interstitial pneumonia.
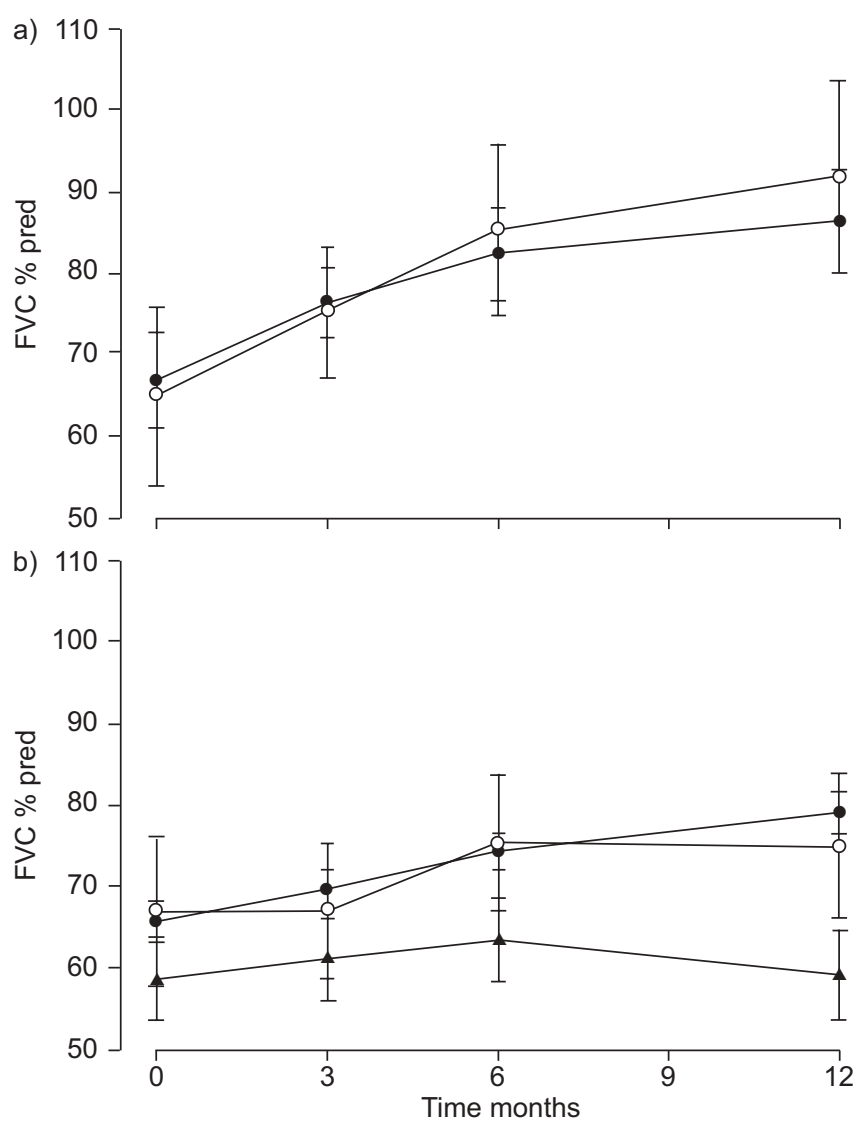

FIGURE 2. The changes in forced vital capacity (FVC) over a 12-month period in patients with a) cellular and b) fibrotic nonspecific interstitial pneumonia (NSIP). One-way repeated measures ANOVA showed a significant difference in FVC at 12 months between survivors and nonsurvivors in patients with fibrotic NSIP $(p=0.042)$.
- : survival; $\bigcirc$ : unrelated death; $\mathbf{\Delta}$ : death.
$47.3 \pm 7.7$ yrs) developed clinical manifestation of CVDs during follow-up. The median (range) interval was variable (21 months (9 months-8 yrs)) and the type of CVDs were also diverse: scleroderma $(n=2)$, polymyositis/dermatomyositis $(n=2)$, rheumatoid arthritis $(n=2)$, mixed connective tissue disease $(n=1)$ and polymyalgia rheumatica $(n=1)$. These patients were younger compared with the remaining idiopathic NSIP patients and rheumatoid factor was more frequently detected in these patients. However, there was no significant difference in sex, baseline pulmonary function or antinuclear antibody positivity between the two groups (see online supplementary material). Among eight patients who later developed CVD, only one had cellular NSIP and died of tuberculous myositis secondary to steroid treatment. Seven patients had fibrotic NSIP and none of them deteriorated progressively. Disease recurred in two patients after the initial improvement. However, the incidence of recurrence was the same as for the patients with continued idiopathic NSIP $(p=1.000)$ and there was no statistically significant difference in the overall prognosis between two groups (see online supplementary material).

\section{DISCUSSION}

The present authors found that the majority $(81 \%)$ of patients with fibrotic NSIP had improved or stable lung function over time after initial treatment. However, even those patients who had initially responded to therapy were frequently hospitalised for respiratory problems with a recurrence rate of $36 \%$ and a disease-related mortality of $30 \%$ in this subgroup. Additionally, a subset of the patients (19\%) deteriorated progressively despite therapy with $69 \%$ mortality among these patients at 5 yrs. The extent of consolidation and ground-glass opacity on initial HRCT was significantly correlated with serial changes of lung function, and the presence of honeycombing predicted poor survival. In patients with cellular NSIP, there 
TABLE 4 Initial high-resolution computed tomography (HRCT) findings of nonspecific interstitial pneumonia (NSIP)

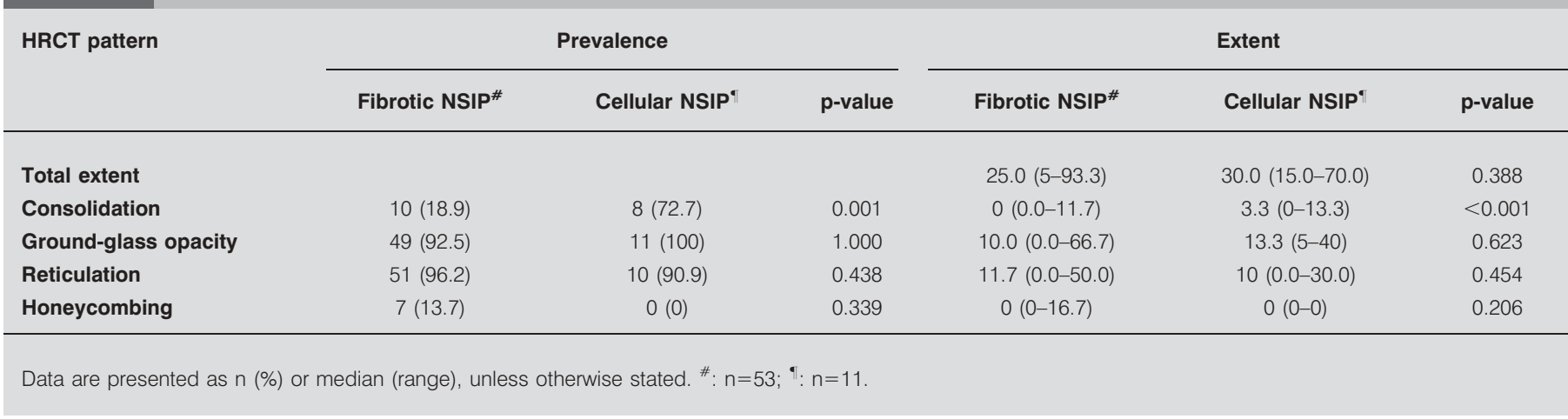

was no hospitalisation for respiratory diseases, although recurrence rate was similar to that of fibrotic NSIP. About $10 \%$ of patients who were initially diagnosed with idiopathic NSIP developed CVD at a later date, although there was no significant difference in clinical presentation except for a younger age and a higher frequency of rheumatoid factor in the initial clinical manifestations.

In some previous reports, the most common HRCT finding in NSIP was ground-glass opacity, and consolidation was less common [11, 16-18], whereas for Asian patients, NAGAI et al. [19] reported that the prevalence of consolidation was $66.7 \%$ in fibrotic NSIP. In the present subjects, the prevalence of groundglass opacity and reticulation was almost $100 \%$ and consolidation was much less prevalent than ground-glass opacity and reticulation. The reason for this discrepancy is not certain; however, the subject number of NAGAI et al. [19] was small (15 patients); the present series was much larger (53 patients) and specifically excluded patients who had a high percentage of consolidation.

It has been reported that initial HRCT findings have prognostic significance in IPF, in that the patients with an atypical pattern for IPF on HRCT had better prognosis than those with typical HRCT pattern for IPF [7, 20]. In NSIP, SCREATON et al. [21] reported that the extent of consolidation on initial HRCT was significantly associated with change in overall extent of parenchymal abnormality on follow-up. The present study also showed that the presence of honeycombing predicts poor survival, and the extent of consolidation and ground-glass opacity correlated with serial changes in lung function in fibrotic NSIP, compatible with the concept of reversibility of these lesions.

Because the presence of honeycombing on initial HRCT was associated with poor survival, the current authors looked at whether these patients may actually represent a subgroup of IPF, either misclassified by sampling error of biopsy or pathologic misinterpretation. The extent of honeycombing was $<5 \%$ in six out of seven patients. Three of them who progressively deteriorated had less honeycombing $(<2 \%)$ than those with initial improvement. Only one patient showed honeycombing more than 5\% (16.7\%); biopsy specimens from this patient were obtained from two different segments of right lower lobe where honeycombing was most prominent on HRCT, and three pathologists diagnosed as fibrotic NSIP

TABLE 5 Correlation between the extent of abnormalities on initial high-resolution computed tomography with pulmonary function in fibrotic nonspecific interstitial pneumonia

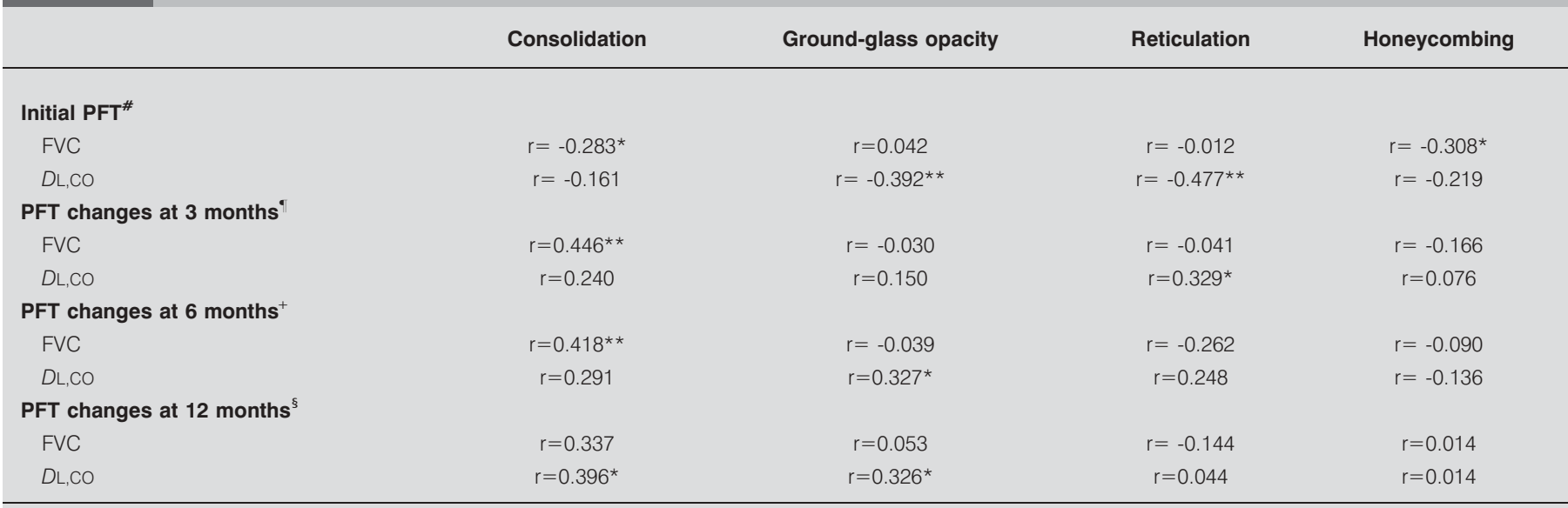

PFT: pulmonary function test; FVC: forced vital capacity; DL,CO: diffusion capacity of the lung for carbon monoxide. ${ }^{\#}: n=53 ;{ }^{\bullet}: n=48 ;{ }^{+}: n=41 ;{ }^{\varsigma}: n=42 .{ }^{*}: p<0.05$ $* *: p<0.01$. 


\begin{tabular}{|c|c|c|c|c|}
\hline \multirow[t]{2}{*}{ TABLE 6} & \multicolumn{4}{|c|}{$\begin{array}{l}\text { Univariate Cox proportional hazard models } \\
\text { predicting survival in patients with fibrotic } \\
\text { nonspecific interstitial pneumonia }\end{array}$} \\
\hline & & Hazard ratio & $95 \% \mathrm{Cl}$ & p-value \\
\hline \multicolumn{2}{|l|}{ Age } & 1.052 & $1.003-1.103$ & 0.037 \\
\hline \multicolumn{2}{|l|}{ Sex } & 0.324 & $0.120-0.872$ & 0.026 \\
\hline \multicolumn{2}{|c|}{ Duration of dyspnoea } & 1.018 & $0.959-1.080$ & 0.569 \\
\hline \multicolumn{2}{|c|}{ Initial resting $\mathrm{Pa}_{2} \mathrm{O}_{2} \mathrm{mmHg}$} & 0.986 & $0.951-1.022$ & 0.429 \\
\hline \multicolumn{2}{|c|}{ Antinuclear antibody } & 0.953 & $0.373-2.847$ & 0.953 \\
\hline \multicolumn{2}{|c|}{ Rheumatoid factor } & 4.447 & $0.586-33.720$ & 0.149 \\
\hline \multicolumn{5}{|c|}{ Initial BAL finding \% } \\
\hline \multicolumn{2}{|c|}{ Macrophage } & 1.001 & $0.972-1.031$ & 0.950 \\
\hline \multicolumn{2}{|c|}{ Lymphocyte } & 0.997 & $0.968-1.028$ & 0.868 \\
\hline \multicolumn{2}{|l|}{ Neutrophil } & 0.995 & $0.942-1.051$ & 0.863 \\
\hline \multicolumn{2}{|l|}{ Eosinophil } & 1.109 & $0.996-1.235$ & 0.060 \\
\hline \multicolumn{5}{|l|}{ Initial PFTs } \\
\hline \multicolumn{2}{|c|}{ FVC \% pred } & 0.963 & $0.928-0.998$ & 0.039 \\
\hline \multicolumn{2}{|c|}{ FEV $1 \%$ pred } & 0.990 & $0.962-1.018$ & 0.481 \\
\hline \multicolumn{2}{|c|}{ DL,CO \% pred } & 0.972 & $0.945-0.999$ & 0.045 \\
\hline \multicolumn{2}{|c|}{ TLC \% pred } & 0.953 & $0.916-0.992$ & 0.020 \\
\hline \multicolumn{2}{|c|}{ Initial dose of prednisolone } & 0.984 & $0.945-1.025$ & 0.434 \\
\hline \multicolumn{2}{|c|}{ Duration of initial treatment } & 0.955 & $0.900-1.014$ & 0.129 \\
\hline \multicolumn{2}{|c|}{ Combination of cytotoxic agent } & 0.692 & $0.156-3.066$ & 0.627 \\
\hline \multicolumn{2}{|c|}{$\begin{array}{l}\text { Duration of high-dose } \\
\text { prednisolone }\end{array}$} & 1.045 & $0.885-1.233$ & 0.606 \\
\hline \multicolumn{5}{|c|}{ Initial 6-min walk test } \\
\hline \multicolumn{2}{|l|}{ Distance } & 0.997 & $0.991-1.002$ & 0.218 \\
\hline \multicolumn{2}{|c|}{ Change in saturation } & 1.128 & $1.030-1.235$ & 0.009 \\
\hline \multicolumn{5}{|l|}{ Initial HRCT } \\
\hline \multicolumn{2}{|c|}{ Ground-glass opacity } & 1.088 & $0.139-8.526$ & 0.936 \\
\hline \multicolumn{2}{|c|}{ Consolidation } & 0.250 & $0.031-1.983$ & 0.189 \\
\hline \multicolumn{2}{|c|}{ Reticular opacity } & 22.090 & $0.000-2029981.1$ & 0.596 \\
\hline \multicolumn{2}{|c|}{ Honeycombing } & 5.582 & $1.605-19.414$ & 0.007 \\
\hline \multicolumn{2}{|c|}{ Respiratory hospitalisation } & 0.126 & $0.036-0.441$ & 0.001 \\
\hline Developmen & of CVD & 0.040 & $0.000-24.349$ & 0.325 \\
\hline
\end{tabular}

Cl: confidence interval; $\mathrm{Pa}_{1} \mathrm{O}_{2}$ : arterial oxygen tension; $\mathrm{BAL}$ : bronchoalveolar lavage; PFT: pulmonary function test; FVC: forced vital capacity; \% pred: \% predicted; FEV1: forced expiratory volume in one second; $D L, C O$ : diffusion capacity of the lung for carbon monoxide; TLC: total lung capacity; HRCT: highresolution computed tomography; CVD: collagen vascular disease. $1 \mathrm{mmHg}=0.133 \mathrm{kPa}$.

without controversy. Furthermore, the patient's lung function was improved after completion of initial treatment. Therefore, the current authors think the possibility of misclassification is very low and that a minor component of honeycombing is not inconsistent with the diagnosis of NSIP.

In studies of fibrotic idiopathic interstitial pneumonias, including fibrotic NSIP and UIP, the 6-month change in FVC has been shown to be an independent risk factor for increased mortality [5, 22]. However, in the present study with fibrotic NSIP only, FVC change at 6 months was not a good predictor, because at 6 months FVC improved in most fibrotic NSIP patients. Even the patients who died with recurrence of disease had improvement of FVC at 6 months. However, FVC changes at 12 months was a good predictor of mortality, with substantially higher mortality in patients with $>10 \%$ deterioration in FVC. The present data seem to confirm those of LATSI et al. [6], although the latter included IPF as well as NSIP patients, and the present data are from purely NSIP. In IPF, FVC decreased steadily and in NSIP most patients improved initially (except a subset of patients who deteriorated steadily, in a similar manner to IPF) but, in nonsurvivors, FVC began to deteriorate after 6 months, making the change in FVC at 12 months a predictor of a poor outcome.

Although the mortality was lower compared to IPF, $76 \%$ of the deaths in this series of NSIP were due to progression of disease in patients whose lung disease recurred or in those who were nonresponders to initial treatment (fig. 1).

Since KATZENSTEIN and Fiorelli [1] first reported the histological features of NSIP in 1994, the NSIP pattern has been found in a variety of nonidiopathic forms of ILD, such as CVD, hypersensitivity pneumonia, lymphoid interstitial pneumonia, and exposure to drugs and occupational or other environmental agents [11]. Recently, this pattern was reported to be the most frequent pathological pattern in interstitial pneumonia associated with polymyositis/dermatomyositis [23], progressive systemic sclerosis [24] and Sjogren's syndrome [25], although the frequency of histopathological patterns in rheumatoid arthritis-associated ILD remains controversial [10, 26 , 27]. Furthermore, SATO et al. [28] reported seven patients with histologically proven NSIP who developed typical CVD 6-35 months after the initial presentation as an idiopathic NSIP. Additionally, KINDER et al. [29] also recently reported that the majority of patients classified as idiopathic NSIP met the definition of undifferentiated connective tissue disease. The current authors also found that eight patients with clinically and histologically proven idiopathic NSIP developed typical CVD, from 9 months to almost 8 yrs after the first presentation. However, no difference was found, except for younger age and higher frequency of rheumatoid factor, between the patients remaining as idiopathic NSIP compared with those who later developed CVD. Therefore, an association with CVD should be searched for vigorously, not only at the time of initial diagnosis but also during the follow-up, in patients with histologically proven NSIP even in the absence of classical systemic involvement at first presentation, especially in younger patients with positive rheumatoid factor. Although there was no NSIP-related death or progressive deterioration in patients with fibrotic NSIP who develop CVD later, that was not a statistically significant risk factor for survival and progressive deterioration. The current authors think that this may be due to the small number of patients and that there remains a possibility that the development of CVD is a favourable prognostic factor for NSIP.

The present study had several limitations. It was a retrospective study performed in a single centre, and several physiological parameters were missing from the follow-up data. However, the majority of the patients were followed up regularly with frequent PFTs. The median follow-up period of the patients was relatively short (53 months) and the range was variable (0.3-181 months). The survival curve of the patients with fibrotic NSIP seems to flatten at 4 yrs after diagnosis (see online supplementary material), in contrast to the report of TRAVIS et al. [4], and there is a possibility that this might be influenced by the 
short and variable follow-up period. However, the median 53 months follow-up was sufficiently long to analyse survival, and 13 patients among 72 with fibrotic NSIP were followed up over 100 months. The treatment regimen and duration were not standardised. However, $82 \%$ of the patients with fibrotic NSIP were treated with corticosteroid and cytotoxic agent (mostly with azathioprine, sometimes switched to cyclophosphamide due to abnormal liver function tests secondary to azathioprine). The other limitation is that about half of the patients had autoantibodies, although no patient had any clinical manifestation either symptoms or signs of CVD on vigorous investigation. The possibility that some of the present patients may still develop CVD after the long-term follow-up still exists. In spite of these limitations, the present study includes the largest number of patients clinico-radiologically and pathologically classified as idiopathic NSIP to date, and provides important information on the clinical course of this disease and its prognostic markers. Further studies of the pathological and radiological features in fibrotic NSIP with longer follow-up will be needed to determine the best predictor in response to treatment and prognosis.

In conclusion, it was observed that, despite a general trend toward improvement or stability in treated patients with idiopathic nonspecific interstitial pneumonia, $\sim 40 \%$ experienced recurrence after the discontinuation of treatment. Furthermore, a subset of the patients with fibrotic nonspecific interstitial pneumonia did not respond to therapy and had a high mortality rate, similar to that in idiopathic pulmonary fibrosis. About $10 \%$ of the patients developed collagen vascular disease during follow-up and they were younger and had higher rheumatoid factor positivity. It was also found that honeycombing on the initial high-resolution computed tomography, initial pulmonary function test and changes in forced vital capacity at 12 months have considerable prognostic value. Cellular nonspecific interstitial pneumonia had much better prognosis than fibrotic nonspecific interstitial pneumonia, although recurrence may occur.

\section{REFERENCES}

1 Katzenstein AL, Fiorelli RF. Nonspecific interstitial pneumonia/fibrosis. Histologic features and clinical significance. Am J Surg Pathol 1994; 18: 136-147.

2 American Thoracic Society/European Respiratory Society International Multidisciplinary Consensus Classification of the Idiopathic Interstitial Pneumonias. Am J Respir Crit Care Med 2002; 165: 277-304.

3 Leslie KO. Historical perspective: a pathologic approach to the classification of idiopathic interstitial pneumonias. Chest 2005; 128: Suppl. 1, 513S-519S.

4 Travis WD, Matsui K, Moss J, Ferrans VJ. Idiopathic nonspecific interstitial pneumonia: prognostic significance of cellular and fibrosing patterns: survival comparison with usual interstitial pneumonia and desquamative interstitial pneumonia. Am J Surg Pathol 2000; 24: 19-33.

5 Jegal Y, Kim DS, Shim TS, et al. Physiology is a stronger predictor of survival than pathology in fibrotic interstitial pneumonia. Am J Respir Crit Care Med 2005; 171: 639-644.

6 Latsi PI, du Bois RM, Nicholson AG, et al. Fibrotic idiopathic interstitial pneumonia: the prognostic value of longitudinal functional trends. Am J Respir Crit Care Med 2003; 168: 531-537.

7 Flaherty KR, Thwaite EL, Kazerooni EA, et al. Radiological versus histological diagnosis in UIP and NSIP: survival implications. Thorax 2003; 58: 143-148.

8 Monaghan H, Wells AU, Colby TV, du Bois RM, Hansell DM, Nicholson AG. Prognostic implications of histologic patterns in multiple surgical lung biopsies from patients with idiopathic interstitial pneumonias. Chest 2004; 125: 522-526.

9 Flaherty KR, Toews GB, Travis WD, et al. Clinical significance of histological classification of idiopathic interstitial pneumonia. Eur Respir J 2002; 19: 275-283.

10 Park JH, Kim DS, Park IN, et al. Prognosis of fibrotic interstitial pneumonia: idiopathic versus collagen vascular disease-related subtypes. Am J Respir Crit Care Med 2007; 175: 705-711.

11 Kim DS, Collard HR, King TE Jr. Classification and natural history of the idiopathic interstitial pneumonias. Proc Am Thorac Soc 2006; 3: 285-292.

12 Kondoh Y, Taniguchi H, Kitaichi M, et al. Acute exacerbation of interstitial pneumonia following surgical lung biopsy. Respir Med 2006; 100: 1753-1759.

13 ATS statement: guidelines for the six-minute walk test. Am J Respir Crit Care Med 2002; 166: 111-117.

14 Kim DS, Paik SH, Lim CM, et al. Value of ICAM-1 expression and soluble ICAM-1 level as a marker of activity in sarcoidosis. Chest 1999; 115: 1059-1065.

15 Akira M, Hamada H, Sakatani M, Kobayashi C, Nishioka M, Yamamoto S. CT findings during phase of accelerated deterioration in patients with idiopathic pulmonary fibrosis. AJR Am J Roentgenol 1997; 168: 79-83.

16 Lynch DA. Fibrotic idiopathic interstitial pneumonia: highresolution computed tomography considerations. Semin Respir Crit Care Med 2003; 24: 365-376.

17 Elliot TL, Lynch DA, Newell JD Jr, et al. High-resolution computed tomography features of nonspecific interstitial pneumonia and usual interstitial pneumonia. J Comput Assist Tomogr 2005; 29: 339-345.

18 Flieder DB, Koss MN. Nonspecific interstitial pneumonia: a provisional category of idiopathic interstitial pneumonia. Curr Opin Pulm Med 2004; 10: 441-446.

19 Nagai S, Kitaichi M, Itoh H, Nishimura K, Izumi $T$, Colby TV. Idiopathic nonspecific interstitial pneumonia/ fibrosis: comparison with idiopathic pulmonary fibrosis and BOOP. Eur Respir J 1998; 12: 1010-1019.

20 Martinez FJ. Idiopathic interstitial pneumonias: usual interstitial pneumonia versus nonspecific interstitial pneumonia. Proc Am Thorac Soc 2006; 3: 81-95.

21 Screaton NJ, Hiorns MP, Lee KS, et al. Serial high resolution CT in non-specific interstitial pneumonia: prognostic value of the initial pattern. Clin Radiol 2005; 60: 96-104.

22 Flaherty KR, Mumford JA, Murray S, et al. Prognostic implications of physiologic and radiographic changes in idiopathic interstitial pneumonia. Am J Respir Crit Care Med 2003; 168: 543-548.

23 Arakawa H, Yamada H, Kurihara Y, et al. Nonspecific interstitial pneumonia associated with polymyositis and dermatomyositis: serial high-resolution CT findings and functional correlation. Chest 2003; 123: 1096-1103. 
24 Kim DS, Yoo B, Lee JS, et al. The major histopathologic pattern of pulmonary fibrosis in scleroderma is nonspecific interstitial pneumonia. Sarcoidosis Vasc Diffuse Lung Dis 2002; 19: 121-127.

25 Yamadori I, Fujita J, Bandoh S, et al. Nonspecific interstitial pneumonia as pulmonary involvement of primary Sjogren's syndrome. Rheumatol Int 2002; 22: 89-92.

26 Lee HK, Kim DS, Yoo B, et al. Histopathologic pattern and clinical features of rheumatoid arthritis-associated interstitial lung disease. Chest 2005; 127: 2019-2027.
27 Yoshinouchi T, Ohtsuki Y, Fujita J, et al. Nonspecific interstitial pneumonia pattern as pulmonary involvement of rheumatoid arthritis. Rheumatol Int 2005; 26: 121-125.

28 Sato T, Fujita J, Yamadori I, et al. Non-specific interstitial pneumonia; as the first clinical presentation of various collagen vascular disorders. Rheumatol Int 2006; 26: 551-555.

29 Kinder BW, Collard HR, Koth L, et al. Idiopathic nonspecific interstitial pneumonia: lung manifestation of undifferentiated connective tissue disease? Am J Respir Crit Care Med 2007; 176: 691-697. 